\title{
Empirical Comparison of Side Channel Analysis Distinguishers on DES in Hardware
}

\author{
Benedikt Gierlichs, Elke De Mulder, Bart Preneel, and Ingrid Verbauwhede \\ K.U. Leuven ESAT/SCD-COSIC and IBBT \\ Kasteelpark Arenberg 10 \\ 3001 Heverlee, Belgium \\ Email: bgierlic, edemulde, preneel, iverbauw@esat.kuleuven.be
}

\begin{abstract}
Side channel analysis attacks exploit the information leakage of a cryptographic device to lay hands on the secret information that is processed. Several statistical means to extract this information have been proposed since the onset of the research area. In this paper we perform a fair empirical comparison of several side channel analysis distinguishers on a hardware implementation of the Data Encryption Standard.
\end{abstract}

\section{INTRODUCTION}

Digital devices and data resources have become a pervasive aspect of our everyday life and data volumes stored or sent over a communication channel are ever increasing. Typically, a combination of several cryptographic techniques is adopted to minimize the damage that unauthorized access to these data could cause. Most of the digital devices handling data nowadays are equipped with embedded security to ensure, for example, data integrity, authentication and confidentiality. Before commercial use, theoretical algorithms are usually submitted to a thorough security investigation and get the label of being black box secure. Nevertheless, the fact that they are eventually implemented in a real circuit in combination with its physical accessibility brings about new security risks. Indeed, measurable physical properties of the implementation give rise to additional sources of information about the secret key used in the device. These additional sources of information are called side channels and the field that makes use of them to extract secret information is called side channel analysis. The first side channel that was exploited in practice is the timing channel [1], followed by the power consumption [2] and the electromagnetic radiation [3], [4]. Side channel analysis attacks are a serious concern because they can easily break implementations of theoretically secure algorithms, if no extra care has been taken to protect the implementation.

Basically, two different subsets of attacks can be distinguished. The first one includes simple side channel analysis which assumes a direct relationship between the secret key and the side channel leakage. A single or very few observations of the side channel leakage are sufficient to derive the secret key. The second type is called differential side channel analysis. In this case, no direct relationship between secret key and leakage is exploitable, and statistical analysis of a larger set of observations is required.

Within the broad range of side channels and types of attacks, we focus on the area of Differential Power Analysis (DPA), which has expanded enormously over the last decade. The interest of the industry and the eager of the academic world to have a thorough command of the problem are certainly factors that account for this development. Three main research streams manifest themselves distinctly: i) research into new statistical models that bear closer resemblance to real leakage, ii) research into new statistical tests for side channel analysis and iii) research that focuses on counteracting the data dependent leakage or rendering the attacks more difficult.

In this work we concentrate on the second research topic, the statistical tests which are referred to as distinguishers. A vast amount of distinguishers has been put forward to extract secret information out of the leakage. The question "Which is the best distinguisher?" receives increasingly more attention but is not straightforward to answer for several reasons. One reason is the complexity of the attack scenarios. Numerous parameters have an impact which makes it difficult to compare attack results. Furthermore, "best" is not an objective notion. One adversary might judge a distinguisher solely by its ability to detect the correct key (accuracy) while another adversary's criterion might be whether the correct key is among the top five candidates (reliability). The distinguishers' performance also varies for different classes of attack targets with diverse leakage behaviour (robustness). Standaert et al. discuss these issues and propose a framework for the empirical comparison of distinguishers in [5] which is put into practice on two software implementations of the Advanced Encryption Standard in [6]. We complement the previous analysis and apply the framework to attacks on a hardware implementation of the Data Encryption Standard (DES). We study the impact of distinguishers on the overall attack efficiency, measured as the number of observations required to achieve a certain success probability, while keeping all other attack parameters fixed.

The paper is structured as follows. A summary of the basics of DPA attacks is given in Sect. II. Section III briefly describes the distinguishers which we compare. Sect. IV reports on the target of the attacks and the actual results are presented in Sect. V. We conclude our work in Sect. VI.

\section{BASICS OF DPA ATTACKS AND NOTATION}

DPA attacks are, in general, possible whenever the power consumption of the device under attack deterministically depends on the data it processes. However, a DPA attack is not as 
easy as measuring the power consumption and "reading off" the secret key due to incomplete knowledge of the adversary (e.g. implementation details), measurement imprecision, noise, etc. Instead, DPA attacks rely on statistical models and tests.

Basic DPA attacks all follow the same procedure, which we summarize while introducing our notation at the same time.

The cryptographic device storing the sought fixed key is sequentially fed with $N$ known input vectors $p_{i}(i=1, \ldots, N)$. A measured power consumption trace over time of a single call to the cryptographic function with input vector $p_{i}$ is denoted as $m_{i}(t)$. We assume the $m_{i}(t)$ to be vectors covering the time span $t=1, \ldots, q$ of the computation. A (first order) attack targets a chosen and sensitive intermediate value of the cryptographic computation that depends both on (a part of) the input vector $p_{i}$ and a small part $k$ of the key. Small means that it must be feasible to run statistics on the power consumption traces for each possible value of $k$.

In order to perform an attack, the adversary needs to be able to predict the side channel leakage (e.g. expected power consumption) of that intermediate value with a statistical model, usually referred to as hypothetical leakage function. The prediction for input $p_{i}$ and a key guess $k^{\prime}$ is denoted by $L_{k^{\prime}, i}$.

The adversary predicts $L_{k^{\prime}, i}$ for all $N$ input vectors and all possible values of $k^{\prime}$. For the correct guess $k^{\prime}=k$, there exists a statistical correlation between the predictions $L_{k, i}$ and the power consumption samples $m_{i}(t)$ at some unknown point in time $t$. The adversary uses a distinguisher to detect this interdependence which reveals both the value of $k$ and the point in time when the targeted intermediate value is computed.

To simplify the description of selected distinguishers, we introduce more notation. Leakage predictions typically map to a small set of values whose nature depends on what exactly is predicted. For now we denote these values by lowercase Greek letters and their ensemble by $\Phi$ but make their meaning more precise in Sect. V-B. Let $\mu_{k^{\prime}, \delta}(t)$ denote the empirical mean of all $m_{i}(t)$ for which $L_{k^{\prime}, i}=\delta$. Similarly, let $\sigma_{k^{\prime}, \delta}^{2}(t)$ denote the sample variance of all $m_{i}(t)$ for which $L_{k^{\prime}, i}=\delta$. Finally, let $N_{k^{\prime}, \delta}$ denote the cardinality of the set of all $m_{i}(t)$ for which $L_{k^{\prime}, i}=\delta$.

\section{THE DISTINGUISHERS}

\section{A. Difference of Means}

The Difference of Means (DoM) test was proposed by Kocher et al. in their seminal paper [2] and thoroughly reviewed in [7]. The basic test divides a set of curves into two sets $S_{\delta}=\left\{m_{i}(t) \mid L_{k^{\prime}, i}=\delta\right\}$ and $S_{\gamma}=\left\{m_{i}(t) \mid L_{k^{\prime}, i}=\right.$ $\gamma \neq \delta\}$ according to the hypothetical leakage values $L_{k^{\prime}, i}$. For example, when focusing on a single bit $L_{k^{\prime}, i} \in\{0,1\}$ one would partition according to whether that bit flips $\left(L_{k^{\prime}, i}=1\right)$ or not $\left(L_{k^{\prime}, i}=0\right)$, because only bit flips are assumed to consume power. Next, both sets of curves are reduced to their empirical means and the difference between the two resulting curves is computed. For the correct hypothesis $k^{\prime}=k$, the predictions $L_{k^{\prime}, i}$ are correct and the partitioning indeed separates curves that are associated to different values of $L_{k^{\prime}, i}$. This results in a peak in the differential curve at the point in time when the targeted operation is computed. For all other hypotheses $k^{\prime} \neq k$, the partitioning is more or less random and the differential curve is flat. The correct value of $k$ can therefore be identified as the one that yields the highest peak in the differential curves. Formally, the DoM distinguisher computes

$$
\Delta_{k^{\prime}}(t)=\mu_{k^{\prime}, \delta}(t)-\mu_{k^{\prime}, \gamma}(t)
$$

for all hypotheses $k^{\prime}$ and selects the best hypothesis $k^{\circ}=$ $\underset{k^{\prime}, t}{\operatorname{argmax}}\left|\Delta_{k^{\prime}}(t)\right|$.

\section{B. T-test}

The T-test [8], [9] is a more elaborate difference of means test that takes the sample variances $\sigma_{k^{\prime}, \delta}^{2}(t)$ and $\sigma_{k^{\prime}, \gamma}^{2}(t)$ in the two sets as well as their cardinalities $N_{k^{\prime}, \delta}$ and $N_{k^{\prime}, \gamma}$ into account. The T-test distinguisher computes

$$
T_{k^{\prime}}(t)=\frac{\mu_{k^{\prime}, \delta}(t)-\mu_{k^{\prime}, \gamma}(t)}{\sqrt{\frac{\sigma_{k^{\prime}, \delta}^{2}(t)}{N_{k^{\prime}, \delta}}+\frac{\sigma_{k^{\prime}, \gamma}^{2}(t)}{N_{k^{\prime}, \gamma}}}}
$$

for each point in time $t$ and all hypotheses $k^{\prime}$ and decides for $k^{\circ}=\underset{k^{\prime}, t}{\operatorname{argmax}}\left|T_{k^{\prime}}(t)\right|$.

\section{Variance Test}

The Variance test (V-test) [6] evaluates the ratio between the sample variance in the set of all curves (denoted by $\sigma^{2}(t)$ ) and the weighted mean of the sample variances in the sets of the partition. The intuition is that the correct key hypothesis minimizes the denominator and thus maximizes the expression. For each $k^{\prime}$ the distinguisher computes

$$
\sigma_{k^{\prime}}^{2}(t)=\frac{\sigma^{2}(t)}{\frac{1}{N} \sum_{\delta \in \Phi} N_{k^{\prime}, \delta} \sigma_{k^{\prime}, \delta}^{2}(t)}
$$

and selects $k^{\circ}=\underset{k^{\prime}, t}{\operatorname{argmax}} \sigma_{k^{\prime}}^{2}(t)$.

\section{Pearson Correlation}

Brier et al. proposed to use the Pearson correlation coefficient in [10]. The distinguisher estimates the coefficient

$$
\rho_{k^{\prime}}(t)=\frac{\operatorname{cov}\left(m_{i=1 \ldots N}(t), L_{k^{\prime}, i=1 \ldots N}\right)}{\sigma\left(m_{i=1 \ldots N}(t)\right) \sigma\left(L_{k^{\prime}, i=1 \ldots N}\right)}
$$

for each $t$ and all key hypotheses. The coefficients are values from the interval $[-1,1]$ that give an indication about the linear fit between the two variables. The distinguisher selects $k^{\circ}=$ $\underset{k^{\prime}, t}{\operatorname{argmax}}\left|\rho_{k^{\prime}}(t)\right|$.

\section{E. Spearman's Rank Correlation}

By using Spearman's rank correlation [11] an attacker does not rely anymore on the linear relationship between the hypothetical leakage values and the measured power consumption. Instead, the test measures the monotonic relationship between two variables. Spearman's rank correlation is a nonparametric or distribution-free rank statistic. The main idea 
of this coefficient is to rank the data before estimating the correlation. If tied ranks exist (which is likely the case), Spearman's coefficient is merely Pearson's $\rho$ of the ranked data. Except for the ranking step, the distinguisher works in the same way.

\section{DES ARChitecture, Measurement Setup AND MEASUREMENTS}

The power consumption traces are made freely available in the light of the "DPA Contest" [12]. The reference measurement set provided by the contest allows for an objective comparison of novel and existing attack techniques while excluding differences in measurement setups and environments between adversaries. In the long run, this initiative aspires to be a first step towards an international benchmarking reference.

The algorithm that has been selected for the competition is the Data Encryption Standard (DES). The architecture is a straightforward parallel implementation where each round of DES is executed in a single clock cycle. The architecture contains two registers: $L R$ and $C D$. The register $L R$ stores the plaintext and the intermediate results of the 16 rounds of DES Similarly, $C D$ has the same objective for the key scheduling. More details about the architecture can be found in [13].

\section{EMPIRICAL EVALUATION}

In this section we describe the comparison criteria applied to the different distinguishers. This is followed by a detailed explanation of the attack procedures before we elaborate on the actual results.

\section{A. Comparison Criteria}

As mentioned before, Standaert et al. propose a framework for the empirical comparison of distinguishers in [5]. To ensure a fair evaluation and following the framework, we apply two criteria. The first-order success rate measures the probability of a distinguisher's best guess being the correct key, i.e. $\operatorname{prob}\left(k^{\circ}=k\right)$. The guessing entropy on the other hand measures the position of the correct key in a list of key hypotheses ranked by a distinguisher. For the DoM test for example, the hypotheses would be listed in decreasing order of $\left|\Delta_{k^{\prime}}(t)\right|$.

\section{B. Attacks}

We aim to attack the intermediate result after the first round of DES. For the purpose of this evaluation, we restrict our attention to the four output bits of the first S-box, which allows to recover 6 out of the 48 bits of the round key. A good attack target in the implementation is the update of the register $L R$, where the four output bits of S-box 1 are stored after a permutation. We assume that the power consumption of the register update is related to the number of bits that flip and thus use the Hamming distance as hypothetical leakage function. More precisely, we predict the hamming distance of the four bits stored in $L R$ before the first round (i.e. the plaintext $p_{i}$ after it passed through the initial permutation of the DES algorithm), denoted by $L R_{0}$, and after the first round under the assumption of $k^{\prime}$, denoted by $L R_{1}$, thus $L_{k^{\prime}, i}=H D\left(L R_{0}, L R_{1}\right) \in\{0, \ldots, 4\}$.

We assume that no more than 350 measurements are required for a successful attack. We can thus use the pool of 81000 measurements available to conduct 230 independent experiments from which we compute average results. For the DoM test and the T-test we have to choose the two subsets of the partition of which we compute the distance. Intuitively, the subsets for which $L_{k^{\prime}, i}$ equals 0 and 4 respectively should yield the largest (and hence easiest to detect) distance. However, using only these two subsets means to discard the majority of the measurements. Using the subsets for which $L_{k^{\prime}, i}$ is smaller/larger than 2 ensures the use of more measurements but yields a smaller distance to detect. Since it is unclear which choice is best we investigate both options and denote them $a$ and $b$ respectively.

\section{Results}

Figure 1 shows the first-order success rates for the distinguishers DoM, T-test, V-test, Pearson's $\rho$ and Spearman's $\rho$ as a function of the number of measurements used for the attack.

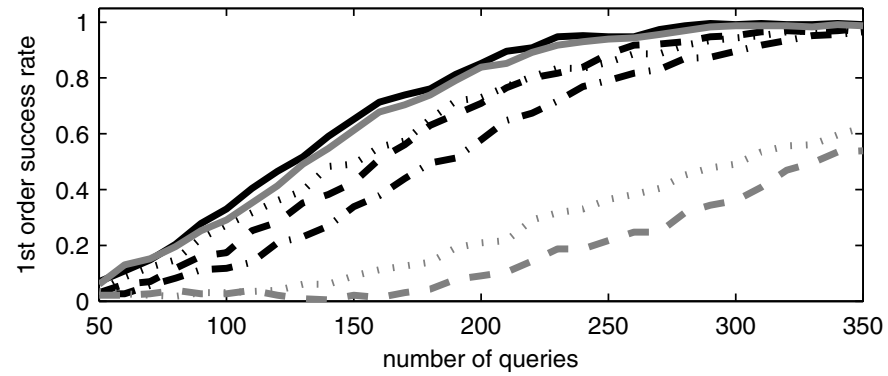

Fig. 1. First-order success rates: DoM (dotted, option $a$ in grey), T-test (dashed, option $a$ in grey), V-test (dash-dotted), Pearson's $\rho$ (solid, black), Spearman's $\rho$ (solid, grey)

According to Fig. 1 both the DoM test and T-test type $a$ show inferior performance. This is easy to understand if we take into account that most of the measurements are actually discarded in this setting. In case of a tailored chosen plaintext attack, the distinguishers would probably move up in the ranking. Excluding DoM type $a$ and T-test type $a$ from the discussion, we can observe that Pearson's $\rho$ performs best, followed by Spearman's $\rho$, the DoM type $b$ and the T-test type $b$. The V-test is positioned last, which indicates that variance is the dominating characteristic of pooled power consumption samples in subsets. In this case, a plausible justification is the algorithmic noise. The small variance caused by the four bits that we do take into account is difficult to detect compared to the changes in the power consumption caused by the 28 key dependent bits that we do not consider. Although we would intuitively assume that T-test type $b$ is superior to DoM type $b$, they actually show equal performance. This corroborates the statement that, in this setting, taking 
the variances in the subsets into account does not yield more exploitable information.

Figure 2 shows the guessing entropies for all distinguishers. Surprisingly, the guessing entropy metric yields quite a differ-

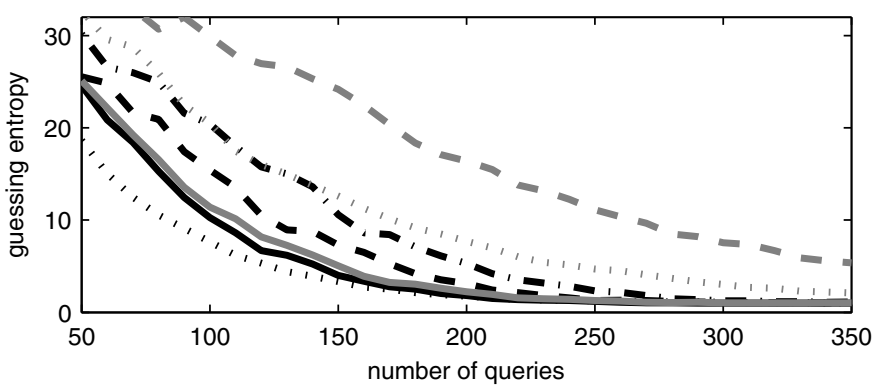

Fig. 2. Guessing entropies: DoM (dotted, option $a$ in grey), T-test (dashed, option $a$ in grey), V-test (dash-dotted), Pearson's $\rho$ (solid, black), Spearman's $\rho$ (solid, grey)

ent picture. The best distinguisher is the DoM type $b$, followed by Pearson's $\rho$, Spearman's $\rho$, T-test type $b$, V-test and DoM type $a$. T-test type $a$ by far ends the enumeration. While in the picture of the success rate Pearson's $\rho$ yields the best success rates, it does not achieve the smallest guessing entropies. This means that the distinguisher is often right, but in the cases when it is wrong it ranks the correct key hypothesis low. We can also see a clear difference between the DoM test type $b$ and the T-test type $b$. Both tests performed similarly in the success rate comparison but with respect to guessing entropy the DoM test type $b$ is better than the T-test type $b$ variant. Hence, both tests are equally good at detecting the correct key but when wrong, the T-test ranks the correct key lower than the DoM test. Again, this can be attributed to the inclusion of the variances into the test statistic. The same variances also affect the performance of the V-test. Concerning the DoM test type $a$ and T-test type $a$, we can say that for the guessing entropy the DoM test does not perform as poorly as it did for the success rate. This means that it ranks the correct key guess in a fairly high position, but because of discarding a lot of the measurements, this guess is rarely put at the highest position. The T-test type $a$ suffers from the same problem as the T-test type $b$ caused by the effect of the variances.

\section{CONCLUSION}

In this paper we perform a fair empirical comparison of side channel distinguishers for DPA attacks on a hardware implementation of DES by means of the first-order success rate and the guessing entropy. Even though only one implementation has been examined in one particular attack scenario, still three fairly general conclusions can be drawn. Firstly, a criterion independent optimal distinguisher does probably not exist. The choice of criterion, in this case the first-order success rate and the guessing entropy, influences the ranking of the different distinguishers remarkably and is an important parameter in the comparison. Secondly, the choice of attacking only one out of eight parallelized S-boxes results into a performance differentiation. The distinguishers that include the variance into their metric perform in general worse than the ones that do not, which indicates that variance is the dominating characteristic of grouped measurements. Thirdly, an attacker bound by the possibility of taking only a limited number of measurements in the random input vector scenario benefits from including all the measurements into the sets of a partitioning distinguisher instead of aiming for a larger distance between the sets. Any other conclusion, e.g. an absolute ranking of the considered distinguishers, is most likely particular to this implementation and attack scenario. Therefore further validation is recommended.

\section{ACKNOWLEDGMENTS}

This work was partially supported by the IAP Programme P6/26 BCRYPT of the Belgian State, by FWO project G.0300.07, by the European Commission under contract number ICT-2007-216676 ECRYPT NoE phase II, by K.U. Leuven-BOF (OT/06/40) and by IWT-Tetra STRES (80138). The information in this document reflects only the author's views, is provided as is and no guarantee or warranty is given that the information is fit for any particular purpose. The user thereof uses the information at its sole risk and liability.

\section{REFERENCES}

[1] P. Kocher, "Timing Attacks on Implementations of Diffie-Hellman, RSA, DSS, and Other Systems," in Advances in Cryptology - CRYPTO '96, ser. LNCS, vol. 1109. Springer, 1996, pp. 104-113.

[2] P. Kocher, J. Jaffe, and B. Jun, "Differential Power Analysis," in Advances in Cryptology - CRYPTO '99, ser. LNCS, vol. 1666. Springer, 1999, pp. 388-397.

[3] K. Gandolfi, C. Mourtel, and F. Olivier, "Electromagnetic Analysis: Concrete Results," in Cryptographic Hardware and Embedded Systems - CHES 2001, ser. LNCS, vol. 2162. Springer, 2001, pp. 251-261.

[4] J.-J. Quisquater and D. Samyde, "ElectroMagnetic Analysis (EMA): Measures and Counter-Measures for Smart Cards," in International Conference on Research in Smart Cards - E-SMART '01, ser. LNCS, vol. 2140. Springer, 2001, pp. 200-210.

[5] F.-X. Standaert, T. G. Malkin, and M. Yung, "A Unified Framework for the Analysis of Side-Channel Key Recovery Attacks," in International Conference on the Theory and Applications of Cryptographic Techniques - Eurocrypt 2009, ser. LNCS, vol. 5479. Springer, 2009, pp. 443-461.

[6] F.-X. Standaert, B. Gierlichs, and I. Verbauwhede, "Partition vs. Comparison Side-Channel Distinguishers," in Information Security and Cryptology - ICISC 2008, ser. LNCS, vol. 5461. Springer, 2008, pp. 253267.

[7] T. Messerges, E. Dabbish, and R. Sloan, "Examining Smart-Card Security under the Threat of Power Analysis Attacks," IEEE Trans. Comput., vol. 51, no. 5, pp. 541-552, 2002.

[8] J.-S. Coron, P. Kocher, and D. Naccache, "Statistics and secret leakage," in Financial Cryptography, ser. LNCS, vol. 1962. Springer, 2000, pp. $157-173$.

[9] B. Gierlichs, K. Lemke-Rust, and C. Paar, "Templates vs. Stochastic Methods." in Cryptographic Hardware and Embedded Systems - CHES 2006, ser. LNCS, vol. 4249. Springer, 2006, pp. 15-29.

[10] E. Brier, C. Clavier, and F. Olivier, "Correlation Power Analysis with a Leakage Model," in Cryptographic Hardware and Embedded Systems CHES 2004, ser. LNCS, vol. 3156. Springer, 2004, pp. 16-29.

[11] L. Batina, B. Gierlichs, and K. Lemke-Rust, "Comparative Evaluation of Rank Correlation based DPA on an AES Prototype Chip," in Information Security Conference - ISC 2008, ser. LNCS, vol. 5222. Springer, 2008, pp. 341-354.

[12] http://www.dpacontest.org/.

[13] S. Guilley, P. Hoogvorst, and R. Pacalet, "A fast pipelined multi-mode DES architecture operating in IP representation," Integr. VLSI J., vol. 40, no. 4, pp. 479-489, 2007. 